\title{
Neuroinfección por Vibrio cholerae no 01/ no 0139 secundaria a la derivación ventriculoperitoneal. Reporte de caso
}

\author{
Julián Andrés Ocampo-Alzate', Nathaly Botero-González', Luz Elena Botero-Rojas², Nelson Morales-Alba ${ }^{3}$
}

\section{RESUMEN}

La infección de la derivación ventriculoperitoneal es una de las complicaciones más frecuentes en este procedimiento. Vibrio cholerae $\mathrm{O} 1$ y O139 es una bacteria gram negativa conocida principalmente por ser la responsable del cólera epidémico. No obstante, existen serotipos no O1/ no O139 capaces de causar afecciones extraintestinales, entre ellas se han reportado casos de neuroinfección. Presentamos el caso de una paciente con 9 meses de edad que posterior a la colocación de una derivación ventriculoperitoneal como tratamiento de hidrocefalia obstructiva congénita, presentó un cuadro de neuroinfección y el síndrome de malfunción valvular; se pudo aislar la Vibrio cholerae no O1/ no O139 en el líquido cefalorraquídeo y en la punta del catéter. Es el primer reporte en la literatura en la que se aísla la Vibrio cholerae no O1/ no O139 en líquido cefalorraquídeo secundario a infección de una derivación ventriculoperitoneal.

\section{PALABRAS CLAVE}

Derivación VentriculoperitoneaI; Infección; Líquido Cefalorraquídeo; Vibrio Cholerae

\section{SUMMARY}

Vibrio cholerae no o1/ no 0139 neuroinfection secondary to ventriculoperitoneal shunt: case report

The infection of the ventriculoperitoneal shunt is one of most frequent complications for this procedure. Vibrio cholera O1 and O139 is a Gram negative bacteria known mainly for being

\footnotetext{
Interno del Hospital Federico Lleras Acosta, Universidad del Tolima, Ibagué, Colombia.

Pediatra del Hospital Federico Lleras Acosta, docente Universidad del Tolima, Ibagué, Colombia.

Neurocirujano del Hospital Federico Lleras Acosta, Ibagué, Colombia.
}

Correspondencia: Julián Andrés 0campo-Alzate; julianandres075@hotmail.com

Recibido: julio 19 de 2018

Aceptado: febrero 12 de 2019

Cómo citar: Ocampo-Alzate JA, Botero-González N, Botero-Rojas LE, Morales-Alba N. Neuroinfección por Vibrio cholerae no 01/ no 0139 secundaria a la derivación ventriculoperitoneal. Reporte de Caso. latreia. 2019 Jul-Sep;32(3):236-242. D0I 10.17533/udea.iatreia.24. 
responsible of the epidemic cholera, however, there are serotypes no O1/ no O139 capable of causing extraintestinal conditions, among them neuroinfection cases have been reported. We present the case of a 9 months old patient who after the placement of a ventriculoperitoneal shunt as treatment for connate obstructive hydrocephalus, presents a neuroinfection condition and valve malfunction syndrome, being able to isolate the Vibrio cholerae no O1/ no O139 in the cerebrospinal fluid and the tip of the catheter. It is the first report in the literature in which the Vibrio cholerae no O1/ no O139 is isolated in the cerebrospinal liquid secondary to an infection from a ventriculoperitoneal shunt.

\section{KEYWORDS}

Cerebrospinal Fluid; Infection; VentriculoperitoneaI Shunt; Vibrio Cholerae

\section{INTRODUCCIÓN}

Una de las principales complicaciones de la derivación ventriculoperitoneal (DVP) son las infecciones, la incidencia reportada es variable, desde el 0,3 al $26 \%$ (1). Diversos factores se han relacionado con la infección de DVP, entre los que destacan los dependientes del paciente (edad, enfermedad principal, hemorragia intraventricular, lesiones cutáneas), con la cirugía (duración, experiencia del neurocirujano, etc.) y con la propia derivación (neurocirugía previa, revisiones o infección previa de la DVP) (2). En el $62-80$ \% de los casos, la infección aparece en el primer mes desde la cirugía, en el $28 \%$ entre el segundo y el duodécimo mes y en el $10 \%$, después del año(2). Los agentes etiológicos que se han aislado con mayor frecuencia en las infecciones secundarias a DVP son Staphylococcus epidermidis, Staphylococcus aureus (60-80\%) y bacilos gramnegativos (10-25\%) (3). Sin embargo, dentro de estos no se ha reportado la participación de Vibrio cholerae.

Existen más de 200 serotipos de la bacteria Vibrio cholerae: Ios serogrupos $\mathrm{O} 1$ y $\mathrm{O} 139$ producen toxinas y causan el cólera clásico con diarrea abundante y acuosa, a lo que se ha conocido como el cólera epidémico (4), los serogrupos no O1/ no O139, generalmente, son cepas no epidémicas notificadas como casos esporádicos (5) que pueden causar infecciones intestinales como la gastroenteritis y extraintestinales como la sepsis, infección del sitio operatorio, neuroinfección y colecistitis (6-8).

\section{REPORTE DEL CASO}

Paciente femenina con 9 meses de vida, con antecedente de hemorragia intraventricular grado IV e hidrocefalia obstructiva congénitarazón por la cual fue tratada previamente con fenestración endoscópica a los 5 meses de vida y una DVP 15 días antes del ingreso a nuestra institución. Los procedimientos fueron realizados sin complicaciones en instituciones hospitalarias del departamento de CaIdas. El acompañante refiere que fue dada de alta luego de la realización de la DVP y se trasladó a su lugar de residencia ubicado en un barrio de baja condición socioeconómica en el municipio de Puerto Salgar, Cundinamarca, donde no se realizaron curaciones a la herida quirúrgica en los siguientes 15 días. Es remitida al servicio de urgencias de un hospital de tercer nivel de complejidad de la ciudad de Ibagué, Tolima, en julio del 2017 por presentar cuadro clínico con un día de evolución, consistente en fiebre cuantificada en $40^{\circ} \mathrm{C}$, un episodio emético, irritabilidad y estatus convulsivo manejado con fenitoína y midazolam en un hospital de menor complejidad. En el examen clínico de ingreso, se registró al paciente consciente, alerta, macrocráneo, con fontanela anterior abombada, herida quirúrogica en la región frontal derecha en buen estado, con salida de líquido cefalorraquídeo (LCR), siogno de sol poniente, pupilas de $2 \mathrm{~mm}$ normorreactivas, el resto del examen físico fue normal; frecuencia cardiaca de 186 latidos por minuto, $37,7^{\circ} \mathrm{C}$ de temperatura, glucometría de $116 \mathrm{mg} / \mathrm{dL}, 10 \mathrm{~kg}$ de peso y talla de $67 \mathrm{~cm}$.

Los resultados de los estudios del laboratorio de ingreso fueron los siguientes: hemoglobina: 10,6 mg/dL, hematocrito: $31 \%$, leucocitos: 4.230 , con $56,3 \%$ de linfocitos y $38,5 \%$ de neutrófilos, proteína $C$ reactiva (PCR): $14,8 \mathrm{mg} / \mathrm{L}$, urocultivo negativo, hemocultivos negativos, el coprocultivo, por cuestiones ajenas a la atención médica no fue procesado. Los hallazgos en las tomas seriadas de LCR se describen en la (Tabla 1).

Se inició el manejo con antibiótico con ceftriaxona $(100 \mathrm{mg} / \mathrm{kg} /$ día $)$ y vancomicina $(15 \mathrm{mg} / \mathrm{kg}$ cada 6 
horas) por LCR compatible con la infección. La tomografía cerebral computarizada (TAC) (Figura 1), tomada en el día 1 de la estancia hospitalaria, evidenció una ventriculomegalia supratentorial e infratentorial, sin signos de paso transependimario de LCR sugestivo de hidrocefalia. Se realizó el retiro de la DVP con hallazgos intraoperatorios de la válvula programable de Hakim con detritos y secreción seropurulenta en su interior y se procedió a la colocación de la ventriculostomía externa, sin complicaciones.

Tabla 1. Evolución del citoquímico de LCR

\begin{tabular}{cccccccccc}
\hline Citoquímico LCR & Día 2 & Día 3 & Día $\mathbf{6}$ & Día 13 & Día 18 & Día 21 & Día 23 & Día 26 & Día 28 \\
Color & $\begin{array}{c}\text { Amarillo } \\
\text { pálido }\end{array}$ & $\begin{array}{c}\text { Amarillo } \\
\text { pálido }\end{array}$ & Amarillo & Amarillo & Blanco & Amarillo & Amarillo & Hemorrágico & Amarillo \\
Aspecto & Lig. turbio & Turbio & Turbio & Turbio & Turbio & Turbio & Turbio & Turbio & Lig. Turbio \\
\hline Xantocromía & - & + & + & + & + & + & + & + & + \\
\hline Glucosa $\mathrm{mg} / \mathrm{dL}$ & 0,30 & 0,70 & 0,40 & 26,3 & 1,10 & 42,9 & 43,9 & 49,9 & 70,6 \\
\hline Proteínas $\mathrm{mg} / \mathrm{dL}$ & 275,60 & 174,8 & 820 & 857,7 & 137,6 & $1.306,3$ & 659,9 & 596,3 & 403,2 \\
\hline Leucocitos $/ \mathrm{mm} 3$ & 228 & 2.450 & 2.850 & 550 & 250 & 43 & 370 & 1.085 & 47 \\
\hline Hematíes $\mathrm{mm}$ m & 3 & 600 & 60 & 750 & 320 & 646 & 14.300 & 21.800 & 20 \\
\hline
\end{tabular}

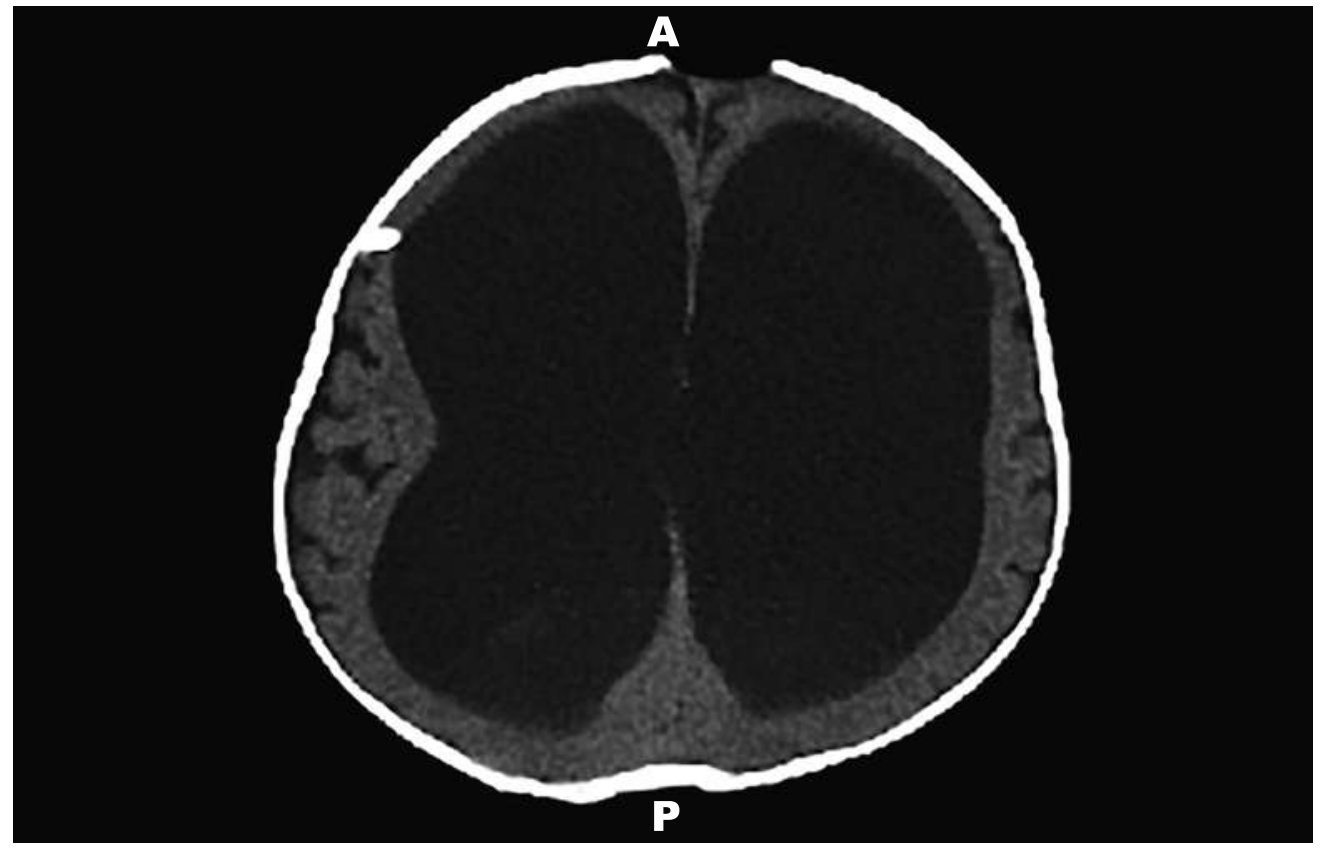

Figura 1. TAC cerebral simple. Ventriculomegalia que sugiere hidrocefalia

El cultivo de LCR reportó un crecimiento en medio de agar MacConkey de bacilos gramnegativos, por lo que se suspendió la ceftriaxona, se inició meropenem (40mg/kg cada 8 horas) y se continuó con vancomicina. Dos días después se tipificó la bacteria como $V i$ brio cholerae, mismo microorganismo reportado en 
el cultivo de punta de catéter de DVP, confirmado días después por el Instituto Nacional de Salud como Vibrio cholerae no O1/ no O139, no toxigénico. Se continuó el manejo instaurado por la sensibilidad de la cepa reportada en el antibiograma, que se realizó por el método de difusión en agar por medio de discos, razón por la cual no se cuenta con la concentración mínima inhibitoria (Tabla 2). Cultivos seriados de LCR posteriores al ajuste antibiótico fueron negativos.

Tabla 2. Antibiograma de Vibrio cholerae no 01/ no 0139

\begin{tabular}{|cc}
\hline Antibiótico & Lectura \\
\hline Cefepime & Sensible \\
\hline Ciprofloxacina & Resistente \\
\hline Doripenem & Sensible \\
\hline Ertapenem & Sensible \\
\hline Ceftazidima & Sensible \\
\hline Ceftriaxona & Sensible \\
\hline Imipenem & Sensible \\
\hline Piperacilina/Tazobactam & Sensible \\
\hline Cefotaxima & Sensible \\
\hline Trimetoprim/Sulfametoxazol & Sensible \\
\hline
\end{tabular}

Pese al manejo antibiótico descrito, la infección intraventricular persiste en los siguientes días, representada por la hipoglucorraquia e hiperproteinorraquia, por lo que se considera la posibilidad de una neuroinfección por otro microorganismo concomitante. Por esto, en el octavo día de la estancia hospitalaria se decide iniciar la vancomicina $20 \mathrm{mg} /$ día durante 7 días por vía intratecal para lograr mayor penetración y concentración del fármaco en el sistema nervioso central. La paciente continuó con deterioro del estado neurológico, hipotonía, parálisis del sexto par craneal representado por estrabismo convergente, múltiples episodios convulsivos manejados con ácido valproico, carbamazepina y fenitoína sin mejoría, y desconexión del medio externo. El día 19 de la estancia hospitalaria presenta ventriculostomía disfuncional, sin drenaje de LCR, por lo que es llevada a ciruogía con hallazgos intraoperatorios de LCR xantocrómico a gran tensión; se retiró la ventriculostomía y se realizó la colocación de una nueva. El día 40 de la estancia se decide retirar la ventriculostomía externa, pues la paciente toleró el cierre de la misma por 48 horas sin presentar signos de hipertensión endocraneana. Por ausencia de signos de esta, sin nuevos episodios convulsivos y cultivos de LCR negativos, se suspendió el manejo antibiótico el día 42 de la estancia; además se consideró al paciente con secuelas neurológicas. Por mal estado neurológico no se establece la DVP definitiva. Se realiza una gastrostomía por el trastorno en la mecánica de la deglución, sin complicaciones; se le da salida con medicación ambulatoria, terapias físicas y control por parte de neuropediatría.

\section{DISCUSIÓN}

La DVP es el tratamiento quirúrogico de elección en pacientes con hidrocefalia comunicante, independientemente de su etiología, por ser muy eficiente $y$ sencilla, pues la cavidad peritoneal es por su alta capacidad de absorción el sitio de drenaje $(9,10)$. Esto ha reducido considerablemente la morbilidad y la mortalidad en los pacientes con hidrocefalia. Sin embargo, las complicaciones asociadas pueden requerir múltiples tratamientos y procedimientos quirúrogicos correctivos durante la vida del paciente.

Entre las complicaciones asociadas a la DVP se encuentra la infección, cuya incidencia reportada es variable desde 0,3 a $26 \%$ (1). Los principales microorganismos que se han identificado como agentes causales de la infección de la DVP son Staphylococcus coagulasa negativo, Staphylococcus aureus, KlebsieIla spp., Escherichia coliy Streptococcus viridans (11).

Las infecciones de las DVP son difíciles de tratary presentan una elevada morbilidad, incluidos el retraso mental y deterioro intelectual. El tratamiento antimicrobiano ocasiona mejoría inicial, incluso curación aparente al erradicar las bacterias superficiales de la biopelícula. Sin embargo, no elimina las bacterias más profundas en estado estacionario, por lo que la recurrencia es la norma si no se retira todo el sistema. Por tanto, hasta la fecha, el tratamiento es médico-quirúroico. En cuanto a los posibles mecanismos de infección, se ha descrito la contaminación durante el acto quirúroico como el más frecuente, seguido por la colonización desde la piel adyacente al shunt y la vía hematógena (1).

En nuestra paciente se aisló, en cultivo de LCR y en punta de catéter de la DVP, el Vibrio cholerae no O1/ 
no O139, situación no descrita previamente en la literatura científica, pues a pesar de que existen reportes de neuroinfección por esta bacteria, no hay antecedentes conocidos de la colonización de este microorganismo secundario a la DVP.

Vibrio cholerae es un bacilo gramnegativo, anaerobio facultativo y oxidasa positivo, que se clasifica por su lipopolisacárido capsular en más de 200 serogrupos, divididos en O1, O139 (responsables de epidemias y pandemias de cólera) y no O1/ no O139 que producen de manera frecuente gastroenteritis, infecciones del oído, de la piel y bacteriemia (12). Sin embargo, se han aislado en otros sitios como en el tracto respiratorio, tracto urinario o LCR (13). No poseen los factores de patogenicidad característicos de la Vibrio cholerae causal del cólera, como la toxina colérica, sino que sintetizan una enterotoxina termoestable que constituye el principal mecanismo asociado a la patogenicidad (14), junto con la producción de proteínas intracelulares responsables de la virulencia y citotoxicidad como la hemolisina, gelatinasa, lecitinasa y elastasa (15).

Existe heterogeneidad en el tratamiento antibiótico en cuanto al agente antimicrobiano, el tiempo y la duración. Las tetraciclinas, azitromicina y ciprofloxacino hacen parte de la primera línea de manejo por su alta efectividad $(16,17)$. Aunque la gastroenteritis secundaria a la Vibrio cholerae no O1/ no O139 es autolimitada, la antibioticoterapia es recomendada en formas complicadas o en pacientes inmunocomprometidos.

La duración de la terapia es materia de discusión, con un rango que está entre 3 y 75 días y un promedio de 14 días. La duración debe ser acorde a la condición clínica del paciente y la gravedad de la infección (abscesos o neuroinfección) (18). Por su baja frecuencia, no existen estudios con evidencia que permitan conducir el manejo en neuroinfección secundaria a esta bacteria. Se han aislado brotes resistentes a múltiples antimicrobianos, entre los que destacan ampicilina, furazolidona, ácido nalidixico, estreptomicina o trimetoprim-sulfametoxazol y una disminución en la sensibilidad al ciprofloxacino (19). Un hecho destacable con el reporte del antibiograma institucional fue el reporte de resistencia al ciprofloxacino, evidenciando la posibilidad de una generación de mecanismos de resistencia bacteriana a un antibiótico usado como primera línea de tratamiento.
El agua de mar parece ser el principal reservorio natural para la Vibrio cholerae no O1/ no O139, ya que requiere pequeñas cantidades de cloruro de sodio para el crecimiento. Sin embaroo, también puede crecer en el agua dulce (20), hecho demostrado con el aislamiento de esta bacteria en aguas superficiales de varios lugares del mundo $(21,22)$. En general, la fuente más común de la infección por Vibrio cholerae no O1/ no O139 es el consumo de mariscos contaminados o crudos, no obstante, la contaminación también puede ocurrir debido a la invasión directa a través de la piel o las heridas erosionadas (4).

Por el tipo de microorganismo aislado y su inusual presencia en las salas de cirugía, consideramos que el mecanismo de infección fue secundario a la manipulación de la herida quirúrgica. En este caso, no se pudo identificar una fuente de infección aparente, aunque la contaminación probablemente ocurrió en los días posteriores a la colocación de la DVP. La madre negó cualquier contacto con el agua de mar o el consumo de alimentos de origen marino, por lo que se desconoce el mecanismo exacto de contaminación.

Es de resaltar que nuestra paciente no presentó diarrea, situación clínica que corrobora lo descrito previamente, pues Vibrio cholerae no O1/ no O139 puede manifestarse con síntomas extraintestinales y sin compromiso intestinal. La clínica presente fue compatible con neuroinfección y con un síndrome de malfunción valvular, hallazgo característico en infecciones por DVP. A pesar del tratamiento antibiótico y quirúrgico recibido, el pronóstico de la paciente fue malo, debido a las características fisiopatológicas $y$ clínicas inherentes a la hemorragia interventricular y a la hidrocefalia obstructiva congénita en asociación al deterioro neurológico secundario al proceso infeccioso descrito $(23,24)$. $(23,24)$. De igual manera, el pronóstico de las neuroinfecciones por microorganismos anaeróbicos suele ser grave y la tasa de mortalidad en niños puede alcanzar el 50 \% (25).

Este reporte de caso evidencia lo inusual de la infección de la DVP por Vibrio cholerae no O1/ no O139 y el riesgo epidemiológico que representa esta bacteria.

\section{CONFLICTOS DE INTERESES}

Ninguno por declarar. 


\section{REFERENCIAS BIBLIOGRÁFICAS}

1. Ram Y, Tina QT. Infections Related to Prosthetic or Artificial Devices. En: Cherry J. Demmler-Harrison G, Kaplan S, et al. Feigin and Cherry's Textbook of Pediatric Infectious Diseases. 7th ed. EIsevier. 2014; 1029-30.

2. Jimenez-Mejias M, Garcia-Cabrera E. Infecciones relacionadas con los sistemas de drenaje de líquido cefalorraquídeo. Enferm Infect Microbiol Clin 2008; 26(4):240-51. DOI 10.1016/S0213-005X(08)72696-X.

3. Gutierrez-Murgas Y, Snowden J. Ventricular shunt infections: Immunopathogenesis and clinical management. J Neuroimmunol. 2014;276(1-2):1-8. DOI 10.1016/j.jneuroim.2014.08.006.

4. Clemens J, Balakrish-Nair G, Ahmed T, Qadri F, Holmgren J. Cholera. Lancet. 2017; 390(10101):39-49. DOI 10.1016/S0140-6736(17)30559-7.

5. Anderson A, Varkey J, Petti C, Liddle R, Frothingham R, Woods C. Non-O1 Vibrio cholerae septicemia: case report, discussion of literature and relevance to bioterrorism. Diagn Microbiol Infect Dis. 2004;49(4):2957. DOI 10.1016/j.diagmicrobio.2004.04.016.

6. West B, Silberman R, Otterson W. Acalculous cholecystitis and septicemia caused by non-O1 Vibrio cholerae: first reported case and review of biliary infections with Vibrio cholerae. Diagn Microbiol Infect Dis. 1998,30(3):187-91.

7. Ismail E, Shafik M, Al-Mutairi G. A case of non-O:1 Vibrio cholerae septicemia with meningitis, cerebral abscess and unilateral hydrocephalus in a preterm baby. Eur J Clin Microbiol Infect Dis. 2001;20(8):598-600.

8. Kerketta J, Chandran-Paul A, Balaji $\nabla$, Kirubakaran C, Jesudason M, Moses P. Non-O1 Vibrio cholerae septicemia and meningitis in a neonate. Indian J Pediatr 2002, 69(10):909-0.

9. Villarejo F, Martínez J. Neurocirugía Pediátrica. Ergon; 2001.

10. Kanev P, Sheehan J. Reflections on shunt infection. Pediatr Neurosurg. 2003;39(6):285-90. DOI 10.1159/000075255.

11. Romero-Garcia M, León-Ramirez A, Carreón-Guerrero J, Romero-Garcia F. Factores de riesgo de infección del sistema de derivación ventriculoperitoneal en pacientes pediátrico. Enf Inf Microbiol. 2014;34(2):59-6.

12. Petsaris O, Nousbaum JB, Quilici ML, Le Coadou G, Payan C, Abalain ML. Non-O1, non-O139
Vibrio cholerae bacteraemia in a cirrhotic patient. J Med Microbiol. 2010; 59(Pt10):1260-2. DOI 10.1099/ jmm.0.021014-0.

13. Lai CC, Liu WL, Chiu YH, Gau SJ, Hsueh PR. Spontaneous bacterial empyema due to non-O1, non-O139 Vibrio cholerae in a cirrhotic patient with hepatoceIlular carcinoma. Diagn Microbiol Infect Dis. 2012; 73(1):84-5. DOI 10.1016/j.diagmicrobio.2012.01.011

14. Farfan M, Miñana D, Fusté MC, Lorén JG. Genetic relationships between clinical and environmental Vibrio cholerae isolates based on multilocus enzyme electrophoresis. Microbiology. 2000;146(Pt10):261326. DOI 10.1099/00221287-146-10-2613.

15. Cabrera Rodríguez LE, Bravo Fariñas L, Ramírez Álvarez M, Llop Hernández A, Fernández Abreu A, Morier Díaz L, et al. Susceptibilidad a los antimicrobianos y factores de virulencia en cepas de $\nabla$. cholerae no-O1 aisladas de pacientes con enfermedad diarreica aguda. Rev Biomed. 2008;19(3):138-44.

16. Fernandez-Abreu A, Bravo-Fariñas L, Rivero-Navea G, Cabrera-Cantelar N, Nuñez-Fernandez S, CruzInfante $\mathrm{Y}$, et al. Determinants of Virulence and Antimicrobial Susceptibility in Non-O1, Non-O139 Vibrio cholerae Isolates. MEDICC Review. 2017; 19(4):21-5.

17. Leibovici-Weissman X, Neuberger A, Bitterman R, Sinclair D, Salam MA, Paul M. Antimicrobial drugs for treating cholera. Cochrane Database Syst Rev. 2014;6:CD008625. DOI 10.1002/14651858.CD008625.pub2.

18. Deshayes S, DaureI C, Cattoir V, Parienti J-J, Quilici M-L, de la Blanchardiere A. Non-O1, non-O139 Vibrio cholerae bacteraemia: case report and literatura review. SprigerPlus. 2015;4:575. DOI 10.1186/s40064-015-1346-3.

19. Ghosh A, Ramamurthy T. Antimicrobials \& cholera: are we stranded? Indian J Med Res. 2011;133(2):225-31.

20. Stypulkowska H, Pancer K, Roszkowiak A. Two unrelated cases of septicemia due to Vibrio cholerae nonO1, non-O139 in Poland, July and August 2006. Euro Surveill. 2006;11(11):E061130.2.

21. Igbinosa E, Okoh A. Emerging Vibrio species: an unending threat to public health in developing countries. Res Microbiol. 2008;159(7-8):495-506. DOI 10.1016/j. resmic.2008.07.001.

22. Couzigou C, Lacombe K, Girard P, Vittecoq D, Meynard J. Non-O:1 and non-O:139 Vibrio cholerae septicemia and pyomyositis in an immunodeficient traveler returning from Tunisia. Travel Med Infect Dis. 2007;5:44-6. 
23. Ayoub M, Laurence F, Arnaud C, Marret S, Vieux R, Aujard $\mathrm{Y}$, et al. Neonatal Infection and 5-year Neurodevelopmental Outcome of Very Preterm Infants. Pediatrics. 2013;132(2):e372-e80. DOI 10.1542/peds.2012-3979.

24. Ros-López B, Jaramillo-Dallimonti A, De MiguelPueyo L, Rodríguez-Barceló S, Domínguez-Páez M, Ibáñez-Botella G, et al. Hemorragia intraventricular del prematuro e hidrocefalia post-hemorrágica. Propuesta de un protocolo de manejo basado en la derivación ventrículo-peritoneal precoz. Neurocirugía. 2009;20:15-24.

25. Brook I. Meningitis and shunt infection caused by anaerobic bacteria in children. Pediatr Neurol. 2002,26(2):99-105. 